\title{
Competencias Pedagógicas que Caracterizan a un Docente Universitario de Excelencia: Un Estudio de Caso que Incorpora la Perspectiva de Docentes y Estudiantes
}

\author{
Verónica A. Villarroel y Daniela V. Bruna \\ Universidad del Desarrollo, Facultad de Psicología, Centro de Investigación y Mejoramiento de la Educación \\ (CIME), Ainavillo 456, Concepción - Chile. (e-mail: vvillarroel@udd.cl; dbrunaj@udd.cl)
}

Recibido Ene. 4, 2017; Aceptado Mar. 8, 2017; Versión final Abr. 20, 2017, Publicado Ago. 2017

\begin{abstract}
Resumen
La presente investigación buscó presentar un modelo de competencias pedagógicas en el ámbito de la Educación Superior. Además, se analizó como esta construcción teórica se relacionaba con la percepción que tienen los estudiantes, docentes y comités académicos, respecto a las competencias que caracterizan a un docente de excelencia. Se realizó un estudio de caso, con un enfoque mixto de investigación, y múltiples estrategias de recolección de datos, en dos sedes de una Universidad Privada chilena. Los resultados mostraron que, si bien existe consenso sobre algunas habilidades que caracterizan a un buen profesor, no existe una mirada homogénea entre los actores. A diferencia de reportes de investigaciones previas, que mencionan que las competencias específicas son las más relevantes, éstas fueron destacadas por los comités académicos, pero no de la misma forma por los estudiantes y profesores. Profesores y estudiantes destacaron más el manejo de conocimiento, comunicación y características personales del educador como las principales competencias de un docente de excelencia.
\end{abstract}

Palabras clave: competencias pedagógicas; docentes universitarios; docentes de excelencia; educación superior

\section{Pedagogical Competencies of University Teachers: A Case Study, Which Incorporates the Perspective of Chilean Teachers and Students}

\begin{abstract}
The aim of this research was to present a pedagogical competencies model in Higher Education. In addition, to analyze how this theoretical construction was related with the perception of students, teachers and academic authorities about the competencies that characterize a teacher of excellence. A case study, with a mix research approach and multiple data collection techniques, was carried out in two campuses of a Chilean private university. The results showed that, although there is consensus of some of the abilities that characterize a good teacher, there is not a homogenous view between the actors. Unlike previous research reports, which mention that specific competences are most relevant, these are consider important for academic committees, but not in the same way for students and teachers. Teachers and students emphasized the management of knowledge, communication skills, and personal characteristics of the educator as the main competences of a teacher of excellence.
\end{abstract}

Keywords: pedagogical competencies; university teachers; teacher of excellence; higher education 


\section{INTRODUCCIÓN}

El sistema educacional chileno ha estado inmerso en procesos de transformación y la educación superior no ha estado exenta de éstos. La formación de alumnos de pregrado se ha convertido en una temática de discusión, especialmente en torno al tipo de aprendizaje y competencias que se espera que los graduados desarrollen en la universidad (Fernández-Cruz y Gijón, 2011; Salgado et al, 2012). En Chile, en los últimos 40 años ha ocurrido un cambio significativo en el número y características de los estudiantes que ingresan a la educación superior. En el pasado, ésta estaba destinada a la formación de la elite. Hasta los años noventa, permaneció como un privilegio destinado a un sector de la sociedad (solo 15 de cada 100 estudiantes continuaban sus estudios hasta la educación superior). Hoy, en cambio, 40 de cada 100 jóvenes acceden a educación superior (Brunner, 2015).

Existen más de 250 instituciones de educación superior que ofrecen cerca de cuatro mil programas de pregrado o carreras (González, 2015). De esta forma ha ocurrido un fenómeno de masificación exponencial. Nunca antes habían existido tantos jóvenes accediendo a formación universitaria y comenzando la vida adulta, como profesionales y técnicos. La educación superior ha incrementado su cobertura, en un $400 \%$, comparado con las estadísticas sobre lo que ocurría 30 años atrás (Ministerio de Educación de Chile, 2013). A medida que el número de jóvenes que accede a la universidad ha aumentado, también lo ha hecho la heterogeneidad entre éstos respecto de sus habilidades, conocimientos y actitudes de entrada. Lo que dificulta el logro de aprendizajes homogéneos y de alta calidad entre los egresados. Esto ha llevado a un cambio y a la creación de mecanismos para garantizar que todos los estudiantes alcancen los resultados de aprendizaje que se esperan en sus profesiones. En definitiva, la educación superior se ha vuelto más compleja (Fernández-Cruz y Gijón, 2011).

En distintas partes del mundo, este interés por el aprendizaje ha derivado en la adopción del modelo de competencias (Juliá, 2012; Zabalza, 2009). Dicho modelo tuvo como propósito relacionar los programas de pregrado universitarios con las necesidades del medio, del sector productivo nacional y la internacionalización. Ésto desde una mirada comprensiva y holística, que transforma el rol del docente y activa el rol del estudiante a fin de recontextualizar los procesos de aprendizaje (Devlin y O'Shea, 2012; Juliá, 2011). Adicionalmente, la atención sobre la calidad de la docencia derivó en esfuerzos que pretendían la renovación de las metodologías educativas. Se buscaba la sustitución del "modelo centrado en la enseñanza" por un "modelo centrado en el aprendizaje" (Biggs y Tang, 2011; Cardona, et al., 2009; Çubukçu, 2012). Este modelo buscaba que el alumno fuera activo en otorgar sentido y significado a los contenidos, para así construir un conocimiento coherente y organizado. En esta nueva aproximación, el docente tiene que modificar y actualizar su forma tradicional de ejercer la docencia. A través del tiempo, la necesidad de transformar las prácticas docentes se ha vuelto más evidente. Los docentes universitarios debieron adoptar un nuevo rol como mediadores o guías del proceso de aprendizaje, dejando de ser el centro del acto educativo (Beaten et al, 2013; Slavich y Zimbardo, 2012). Por otra parte, las instituciones educativas se vieron exigidas a investigar e identificar cuáles eran las competencias docentes requeridas que propician aprendizajes profundos en sus estudiantes.

El concepto de competencia se utilizó por primera vez en los años 70 , como resultado de investigaciones enfocadas en identificar las variables que permitían explicar el desempeño en el trabajo. Se refieren a un conjunto de conocimientos, habilidades y actitudes que el ser humano aplica para aprender, adaptarse y desempeñarse en el mundo (Camargo y Pardo, 2008). En el contexto laboral, las competencias constituyen un complejo conjunto de comportamientos que evidencian la capacidad profesional para usar armónicamente sus conocimientos, experiencias, habilidades, disposiciones, actitudes y valores para abordar, resolver o actuar frente a situaciones en el mundo personal, profesional, cívico y social (Camperos, 2007). La competencia corresponde a un saber-hacer en contexto, donde se movilizan recursos cognitivos, integrando conocimientos, habilidades y valores para enfrentarse a distintas situaciones del quehacer en el ámbito profesional (Perrenoud, 2004).

En el caso de docentes universitarios, el estudio de las competencias pedagógicas se ha desarrollado internacionalmente con el objetivo de generar perfiles docentes, que permitan guiar el entrenamiento/capacitación y evaluación del profesorado. Enseñar en la universidad actual requiere de conocimientos, habilidades y actitudes diferenciadas de las que se necesitaban 20 años atrás. Diversos tipos de modelos respecto de estas competencias han sido creados como resultado de numerosas investigaciones, como son los propuestos por distintos autores como: Galvis (2007), Postareff y LindblomYlänne (2008), Zabalza (2009), Gairín (2011) y Torra et al. (2012), que serán descritos más adelante.

A partir de la revisión de la literatura, en este artículo se presenta un modelo de competencias docentes que incorpora, comprensivamente, las distintas contribuciones que se han hecho en educación superior en este tema. Junto a ello, se analiza cómo esta construcción teórica se relaciona con la percepción que, en la 
práctica, los estudiantes, docentes y equipos académicos tienen respecto a lo que caracteriza un docente de excelencia. Para lograrlo, se realizó un estudio de caso, donde se evaluó si las competencias docentes, que la literatura plantea como requeridas en educación superior, están presentes en un conjunto de profesores evaluados en el nivel de "excelencia" por sus alumnos y equipos directivos en una universidad privada en Chile.

\section{ANTECEDENTES SOBRE COMPETENCIAS DOCENTES}

Han existido distintos estudios destinados a identificar y validar un perfil de competencias del docente universitario, desarrollando modelos que permiten evaluarlo (Abadía et al, 2015; Zabalza, 2009). Galvis (2007) distinguió cuatro competencias del docente universitario, las que llamó: intelectuales, inter e intrapersonales, sociales y profesionales. Las Competencias Intelectuales se vincularían al "conocer", como son dominar conceptos y teorías actualizadas sobre las disciplinas educativas y de su especialidad, poseer una cultura general propia de la educación superior y conocer técnicas de recolección de información. Las Competencias Inter e Intrapersonales se relacionan con el "ser", como son, la apertura hacia las nuevas experiencias, la responsabilidad de sus acciones, la habilidad de adaptarse a los cambios, y de mostrar consistencia. Las Competencias Sociales están asociadas con la habilidad de "convivir con otros", lo que implica practicar la tolerancia, establecer relaciones de diálogo a nivel interpersonal e institucional, y respetar el pensamiento divergente. Finalmente están las Competencias Profesionales que se relacionan con el "hacer", como son, el planificar y evaluar situaciones de aprendizaje significativo, manejar técnicas de trabajo grupal, y aplicar metodologías de evaluación activas que promuevan el aprendizaje. Postareff y Lindblom- Ylänne (2008), conceptualizan la enseñanza centrada en el aprendizaje, en la educación superior, a través de cuatro dimensiones: a) proceso de enseñanza (planificación de la enseñanza, prácticas de enseñanza y estrategias de evaluación), b) ambiente de aprendizaje (rol del docente, rol del estudiante, interacción y atmósfera), c) concepto de aprendizaje; y finalmente d) desarrollo pedagógico (desarrollo de la propia forma de enseñar y consciencia reflexiva sobre la pedagogía).

En la primera dimensión, "Proceso de Enseñanza", la Planificación incorpora las necesidades de los estudiantes, por lo que no es algo rígido, ya que, deja espacio para realizar cambios a partir del desarrollo del curso. Las Prácticas de Enseñanza muestran que el conocimiento es co-construido en conjunto con los estudiantes, y que los docentes están conscientes de que los estudiantes tienen distintas formas de aprender, por lo que utilizan distintos métodos de enseñanza. Las estrategias de Evaluación están directamente dirigidas a medir la comprensión profunda del fenómeno a través de distintos tipos de estrategias de evaluación (Postareff y Lindblom- Ylänne, 2008). En la segunda dimensión, "Ambiente de Aprendizaje", el Rol del Docente es el de un facilitador que muestra una actitud positiva hacia la enseñanza, manteniendo una relación equivalente con cada uno de los estudiantes, motivándolos a ser críticos y activos. La Interacción entre docente-estudiante es lo que construye el conocimiento. La Atmósfera de la clase apoya el aprendizaje, siendo un ambiente que provee seguridad, donde es fácil preguntar, opinar y pedir ayuda (Postareff y Lindblom- Ylänne, 2008). Finalmente, en la tercera dimensión, "Conceptualización del Aprendizaje", el docente comprende el acto de aprender como la construcción de los propios puntos de vista frente a un fenómeno, incorporando la aplicación del conocimiento, el desarrollo de distintas perspectivas, el pensamiento crítico y comprensión profunda. Finalmente, el Desarrollo Pedagógico implica para el docente Desarrollar una Forma Propia de Enseñar y una Conciencia Reflexiva acerca de su propia pedagogía y habilidades docentes (Postareff y Lindblom- Ylänne, 2008).

En España, Zabalza (2009) enumeró nueve cualidades del docente de excelencia: a) Planificar el proceso de enseñanza y aprendizaje, b) Seleccionar y presentar, adecuadamente, los contenidos disciplinares, c) Ofrecer información e explicaciones comprensibles, d) Contar con alfabetización tecnológica y manejo didáctico de las TICs, e) Gestionar las metodologías de trabajo didáctico y las tareas de aprendizaje, f) Relacionarse constructivamente con los alumnos, g) Reflexionar e investigar sobre la enseñanza e i) Implicarse institucionalmente. El autor menciona que el desarrollo de competencias tecnológicas en la enseñanza de los docentes, es una oportunidad de transformar la docencia universitaria y la modalidad de enseñanza y aprendizaje. Según Ortega (2010), el Área Europea de Educación Superior (AEES) también ha identificado ocho competencias del perfil docente. Estas son: a) competencias cognitivas (el conocimiento en un nivel disciplinar y pedagógico), b) competencias metacognitivas (pensamiento crítico, autocrítico y reflexivo), c) competencias culturales y contextuales (conocimientos del entorno y de la organización), d) competencias comunicativas (lenguaje científico verbal y escrito), e) competencias sociales (cooperación, empatía y trabajo en equipo), f) competencias de gestión (gestión, organización y planificación), g) habilidades tecnológicas (aprendizaje, investigación y uso de las TIC), h) competencias de investigación (búsqueda de información sobre metodologías y recursos).

Gairín (2011), propuso que, para que un buen proceso de docencia se lleve a cabo se requiere, el desarrollo de cuatro competencias: técnicas, metodológicas, sociales y personales. Las Competencias Técnicas 
refieren al saber relacionado con el quehacer pedagógico, incluyendo el conocimiento de la disciplina que se enseña y del currículum. Las Competencias Metodológicas son aquellas que vinculan el conocimiento con la realidad de los estudiantes, incorporando las experiencias de los estudiantes y la interacción docenteestudiante durante el proceso de enseñanza. Las Competencias Sociales implican la habilidad para comprender y trabajar con otros, usando indicadores como, actuar bajo criterios acordados colectivamente y participando en la evaluación del proyecto educativo. Finalmente, las Competencias Personales, corresponden a la ética profesional y la responsabilidad en el ejercicio de la profesión. Estas competencias permiten a los docentes desarrollar funciones didácticas (planificación, enseñanza y evaluación), tutoriales (acompañamiento a los estudiantes), de extensión (vinculación con el medio y redes), y la formación constante e innovación en su profesión.

En una línea similar, Torra, De Corral y otros 17 autores (2012) realizaron un estudio en ocho universidades catalanas donde 64 investigadores validaron un modelo de perfil de competencias del docente universitario. Las competencias identificadas fueron: a) Competencia Comunicativa, que incluye explicar con claridad y convicción, facilitando la comprensión de contenidos por parte de los estudiantes, b) Competencia Interpersonal, que involucra promover la motivación, clima de empatía y tolerancia con los estudiantes, c) Competencia Metodológica, relacionada con la coherencia entre los objetivos y los métodos utilizados para la enseñanza y la evaluación, d) Competencia de Planificación y Gestión de la Docencia, que está más ligada al diseño del proceso de enseñanza-aprendizaje, d) Competencia de Innovación, que incluye la reflexión e indagación sobre la propia docencia, d) Competencia de Trabajo en Equipo, donde se gestionan equipos docentes, distribuyendo funciones y potenciando su eficacia y eficiencia.

\section{LA PROPUESTA DE COMPETENCIAS DOCENTES}

A partir de la literatura revisada, se observan competencias comunes a distintos modelos o perfiles propuestos. Por ejemplo, aparece: a) competencias específicas del proceso de enseñanza aprendizaje, como son la competencia profesionales e intelectuales de Galvis (2007), todas las de Postareff (2008), 4 de las 9 señaladas por Zabalza (2009), las cognitivas y de gestión planteadas por la AEES (Ortega, 2010), las técnicas y metodológicas de Gairín (2011), y las comunicativas y metodológicas del estudio de Torra y otros autores (2012), b) competencias interpersonales, como son las Inter e Intrapersonales y sociales en Galvis (20017), Relación con los alumnos en Zabalza (2009), Sociales y Personales en Gairín (2011) y las Interpersonales de Torra y otros autores (2012), c) competencias de investigación como lo señala Gavis (2007) en las competencias intelectuales, Investigación de AEES (Ortega, 2010) e Innovación en Torra y otros autores (2012), en menor medida, d) competencias de comunicación (Ortega, 2010; Torra et al, 2012; Zabalza, 2009) y e) manejo de tecnologías de la información (Ortega, 2010; Zabalza, 2009). Considerando estas competencias comunes, en este artículo se realiza una propuesta de un Perfil de Competencias Docentes que sintetiza las competencias requeridas en la educación universitaria actual en tres dimensiones de competencias: Básicas, Específicas y Transversales. Estas tres dimensiones derivan de conceptualizaciones previas, pero que muestran una reorganización distinta de las mismas. A continuación se describe cada dimensión y las competencias asociadas a ellas.

Las Competencias Básicas corresponderían al conjunto de habilidades, conocimientos y actitudes propias de la labor de un profesor, y que conforman los requisitos o las condiciones mínimas necesarias para el ejercicio de la docencia, pero, por sí solas no dan cuenta de un desempeño destacado o de excelencia. Tienen relación con el conocimiento de las materias que se enseñan, la habilidad de expresarse y dar a entender sus ideas, teorías y conceptos, la capacidad de trabajar junto a otros docentes, mostrar responsabilidad y compromiso con el quehacer y ética del trabajo docente. Involucran competencias: a) Cognitivas asociadas al conocimiento de la disciplina (Cardona et al 2011; Casero, 2008; Ortega, 2010; Galvis, 2007), b) Sociales asociadas al trabajo en equipo y la colaboración con otros (Cano, 2005, Cardona, et al., 2009; Ortega, 2010; Galvis, 2007; Zabalza, 2009), c) Comunicativas referidas la capacidad de expresar y explicarse adecuadamente a nivel oral y escrito (Cano, 2005, Cardona, et al., 2009; Monereo y Domínguez, 2014; Ortega, 2010), d) Tecnológicas ligadas al manejo de las tecnologías de la información (Cano, 2005; Ortega, 2010, Perrenoud, 2004; Zabalza, 2009), y e) Personales como son el compromiso y ética profesional (Cardona, et al., 2009; Casero, 2008; Comellas, 2002, Elton, 1996; Gairín, 2011; Monereo y Domínguez, 2014; Perrenoud, 2004).

Las Competencias Específicas se refieren a las que son parte fundamental del proceso de enseñanza aprendizaje y distinguen a un docente cuya enseñanza está centrada en el aprendizaje de los estudiantes (Mcabe y O'Connor, 2014), buscando identificar y comprender cuáles son las mejores estrategias de enseñanza y evaluación según los resultados de aprendizaje que se quieren desarrollar, los contenidos que se aprenden y las características de los alumnos a quienes enseña (Cid et al, 2012; Moreno, 2016; Torelló, 2012). Éstas involucran competencias de: a) Planificación y Organización referidas a cómo se diseña el proceso de enseñanza-aprendizaje, respetando lo que se debe enseñar en un curso, los resultados de 
aprendizajes que los estudiantes deben lograr y las habilidades de entrada que ellos traen para alcanzarlas (Alvarez-Rojo, et al., 2011; Camargo y Pardo, 2008; Cano, 2005; Comellas, 2002; Elton, 1996; Ghedin, 2008; Monereo y Domínguez, 2014; Ortega, 2010; Perrenoud, 2004; Zabalza, 2009), b) Didácticas entendidas como la selección de estrategias y metodologías de enseñanza que permitirán lograr las metas del curso, considerando las características y necesidades de los estudiantes (Alvarez-Rojo, et al., 2011, Casero, 2008; Gairín, 2011; Morán et al, 2015; Perrenoud, 2004; Postareff y Lindblom- Ylänne, 2008), y c) Diseño e Implementación de Metodologías de Evaluación con que se espera medir y valorar el aprendizaje de los estudiantes, utilizando distintas estrategias que evalúen la construcción del conocimiento y desarrollo de habilidades de orden superior, entregándoles feedback a los estudiantes de sus resultados (Alvarez-Rojo et al, 2011; Camargo y Pardo, 2008; Casero, 2008; Elton, 1966; Morán et al, 2015).

Las Competencias Transversales son aquellas entregan un valor agregado del proceso de enseñanza y aprendizaje. Funcionan como factor protector del rol del docente, su interacción con los estudiantes y el logro de los aprendizajes esperados. Tiene relación con la capacidad metacognitiva, autocrítica y reflexiva del docente, lo que le permite ponerse en el lugar de otros, vincularse adecuadamente con los alumnos, cuestionar y mejorar sus prácticas docentes. Estas competencias influyen en la efectividad y satisfacción de los estudiantes y docentes con el proceso de enseñanza-aprendizaje. Involucran competencias ligadas a: a) Mantener un clima de aula positivo (Cardona, et al., 2009; Camargo y otros autores, 2008; Casero, 2008; Postareff y Lindblom- Ylänne, 2008; Zabalza, 2009), y b) Investigar y reflexionar sobre sus propias practicas pedagógicas (Alvarez-Rojo et al, 2011; Cardona et al, 2009; Camargo y Pardo, 2008; Elton, 1996; Perrenoud, 2004; Zabalza, 2009). La figura 1, resume este modelo recién presentado.

Este perfil fue elaborado con el fin sistematizar modelos presentes en la literatura, como también de contrastar el modelo teórico con nuestra realidad nacional a través de un estudio de caso. El objetivo fue determinar si las competencias sistematizadas en el modelo recién presentado, se encuentran presentes en docentes universitarios calificados en el rango de excelencia, por alumnos y directivos de una universidad privada de Chile. La pregunta de investigación que orienta este estudio fue: ¿los docentes de esta universidad, evaluados por sus alumnos y directivos en la categoría de excelencia, presentan las competencias docentes señaladas los modelos y perfiles expuestos en la literatura especializada en este tema?

\section{MÉTODO}

Esta sección se presenta en tres subsecciones: en la primera se explica y detalla el diseño de la investigación, luego se describe el tipo de participantes indicando la cantidad y tipo de dichos participantes, para luego explicar las técnicas de recolección de la información, y finalmente se detalla el procedimiento seguido para la recolección y análisis de datos.

\section{Diseño}

El diseño de la investigación corresponde a un estudio de caso, donde a través de una metodología de investigación mixta se analiza profundamente el objeto de estudio (competencias docentes presentes en docentes universitarios de excelencia), respondiendo a la pregunta de investigación. Utilizó como estrategia principal un enfoque metodológico mixto o multi-método, que simboliza una tercera vía de investigación en ciencias sociales. Estos métodos representan un conjunto de procesos sistemáticos, empíricos y críticos de investigación. Implican la recolección y el análisis de datos cuantitativos y cualitativos, así como la integración y discusión conjunta para realizar inferencias de la información recabada y lograr mayor comprensión del fenómeno en estudio. Se optó por este método debido a la naturaleza compleja del fenómeno, construido por realidades objetivas y subjetivas. Para capturar estas realidades se utilizan, justamente, diseños multimodales (Hernández-Sampieri et al, 2014).

Es un estudio que involucra integración y conversión de datos. Esto implica que un tipo de datos es convertido en otro (cualificar datos cuantitativos o cuantificar datos cualitativos). Luego se analizan e integran ambos conjuntos de datos bajo el análisis cuantitativo y cualitativo para dar respuesta al problema de investigación. Para la rama cuantitativa se buscó clasificar las competencias presentadas por los docentes al momento de ser observados y jerarquizar las competencias docentes según la encuesta realizada a ellos. Para la rama cualitativa se llevó a cabo análisis de contenido deductivo de las entrevistas (a docentes seleccionados y equipos de gestión académica) y focus group (a los alumnos de las carreras), con el fin de indagar su percepción respecto a las competencias docentes y a las características esperadas en un docente de excelencia. 


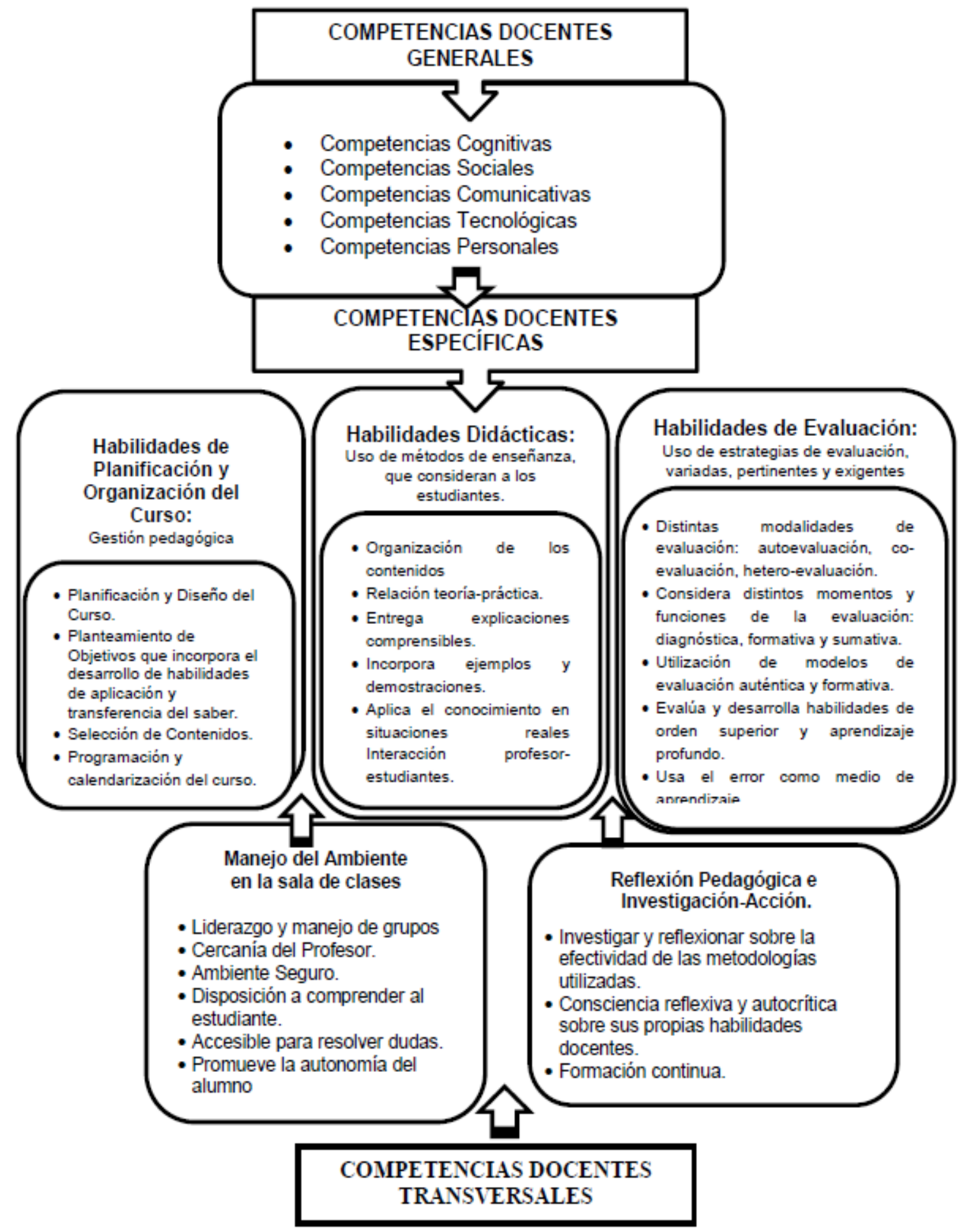

Fig. 1: Propuesta de modelo de competencias docentes

\section{Participantes}

La muestra fue seleccionada intencionalmente con el objetivo de acceder a docentes que han sido evaluados por sus alumnos y equipos de gestión académica en la categoría de excelencia. De esta forma, se utilizó una estrategia opinática, la cual consiste en la selección de los informantes siguiendo criterios estratégicos en función de los objetivos del estudio como: pertinencia, adecuación, conveniencia, oportunidad y disponibilidad. En primera instancia, fueron los directores de carrera, junto a sus equipos de gestión académica, quiénes entregaron una lista con el nombre de los 3 profesores que, según la percepción de los alumnos como la de ellos, se caracterizaban por su excelencia docente. Para su selección utilizaron como criterio la evaluación docente que entregan los alumnos y criterios propios de la facultad, que son más extensamente descritos en el apartado del procedimiento. 
Luego de invitar a los 30 docentes sugeridos, aceptaron participar 26 de ellos. Los profesores pertenecían a 10 programas de pregrado de la Universidad del Desarrollo, 14 de la sede de Concepción y 12 de la sede de Santiago. Las características de los docentes participantes pueden resumirse en: 14 mujeres y 12 hombres, promedio de edad era de 41 años, el promedio de experiencia docente fue de 14.26 años, con un rango mínimo de 6 y máximo de 26 años. Respecto de su nivel de formación académica, 24 docentes contaban con grado de magister y 3 de ellos también contaban con el grado de doctor. Los profesores realizaban clases en distintos niveles: 10 de ellos impartían docencia en primer y segundo año, 8 en tercer y cuarto año, y 8 del quinto año.

Las carreras que colaboraron en el estudio fueron: Ingeniería Civil Industrial, Ingeniería Comercial, Derecho, Odontología, Enfermería, Psicología, Periodismo, Pedagogía Básica, Diseño y Arquitectura. De todos los docentes participantes, 25 fueron observados, 26 entrevistados y 20 encuestados. También participaron 6 alumnos por carrera en los 8 focus group realizados (48 alumnos en total) y fueron entrevistados todos los equipos de gestión académica (generalmente compuestos, como mínimo por: director/a, coordinador/a académico y algún coordinador/a de ciclo) de las carreras involucradas. La Tabla 1 resume esta información:

Tabla 1: Frecuencia de participantes por carrera y por técnica de recolección de datos

\begin{tabular}{|c|c|c|c|c|c|c|}
\hline Sede & Carrera & $\begin{array}{c}N^{\circ} \\
\text { Observación }\end{array}$ & $\begin{array}{l}N^{\circ} \text { Docentes } \\
\text { Entrevistados }\end{array}$ & $\begin{array}{l}N^{\circ} \text { Docentes } \\
\text { encuestados }\end{array}$ & $\begin{array}{l}N^{\circ} \text { Focus } \\
\text { Group } \\
\text { Alumnos }\end{array}$ & $\begin{array}{c}\text { Entrevista equipos } \\
\text { de Gestión } \\
\text { Académica }\end{array}$ \\
\hline \multirow{6}{*}{ Concepción } & $\begin{array}{l}\text { Ingeniería } \\
\text { Civil } \\
\text { Industrial }\end{array}$ & 3 & 2 & 2 & 0 & 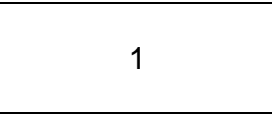 \\
\hline & $\begin{array}{l}\text { Ingeniería } \\
\text { Comercial }\end{array}$ & 2 & 3 & 2 & 1 & 1 \\
\hline & Derecho & 2 & 2 & 2 & 0 & 1 \\
\hline & Odontología & 1 & 2 & 2 & 1 & 1 \\
\hline & Enfermería & 2 & 2 & 2 & 1 & 1 \\
\hline & Psicología & 3 & 3 & 2 & 1 & 1 \\
\hline \multirow{4}{*}{ Santiago } & Periodismo & 3 & 3 & 2 & 1 & 1 \\
\hline & Pedagogía & 3 & 3 & 2 & 1 & 1 \\
\hline & Diseño & 3 & 3 & 2 & 1 & 1 \\
\hline & Arquitectura & 3 & 3 & 2 & 1 & 1 \\
\hline \multicolumn{2}{|l|}{ Total } & 25 & 26 & 20 & 8 (48 alumnos) & 10 \\
\hline
\end{tabular}

\section{Técnicas de recolección de la información}

A partir de la literatura revisada, se decidió utilizar diversas técnicas de recolección de información (observación, entrevista, encuesta, focus group), que involucrarán distintas perspectivas o miradas (desde los alumnos, comités académicos y propio profesor de excelencia) sobre el desempeño docente de un profesor destacado (Tejedor, 2012). Se construyeron las pautas de observación, entrevista y focus group que evalúan el modelo de competencias docentes antes planteado. Cada una de las pautas fue sometida a un proceso de validación por 4 jueces externos. Para estimar la concordancia entre los jueces, se utilizó Kappa de Fleiss, que permite trabajar con más de dos jueces. Se obtuvo un resultado de $\mathrm{Kf}=0.76$ para la pauta de observación, $\mathrm{Kf}=0.89$ para la pauta de entrevista del profesor de excelencia, $\mathrm{Kf}=0.90$ para la pauta de entrevista del equipo de gestión académica, $\mathrm{Kf}=0.81$ para la pauta de focus group, y $\mathrm{Kf}=0.78$ para el cuestionario del profesor de excelencia. Los índices de estas pautas de recolección de información son calificados en el rango de bueno. Esto implica que existe una adecuada concordancia entre los jueces que evaluaron cada pauta.

Las técnicas de recolección de datos utilizadas fueron: 1) Observación de las prácticas pedagógicas usadas por los docentes en el aula a través de una pauta estructurada; 2) Cuestionario para indagar sobre la relevancia que el docente otorga a las distintas competencias docentes que forman parte del modelo teórico testeado; 3) Entrevista en profundidad a los docentes para examinar su percepción respecto a sus competencias como docente y a las competencias en un docente de excelencia, en los distintos niveles de formación de pregrado; 4) Focus Group a estudiantes para acceder a la percepción de los alumnos respecto de las competencias en un docente de excelencia y las distinciones entre niveles (primer y segundo año, tercero y cuarto año, y quinto año); y 5) Entrevista a equipos de gestión académica para indagar respecto a las competencias que caracterizan a los docentes de excelencia seleccionados por ellos.

Todos los instrumentos de recolección utilizados midieron las competencias docentes del modelo teórico construido a partir de la literatura, y presentado anteriormente. Se indagó sobre cada una de las tres 
dimensiones y las competencias asociadas a ellas. En el caso de las entrevistas, éstas fueron realizadas por profesionales psicólogos, con más de 5 años de experiencia en docencia universitaria y con una formación académica en investigación cualitativa. Una persona entrevistó a cada docente utilizando una pauta semi-estructurada. A modo de ejemplo, se presenta el guión de la entrevista al docente de excelencia. La misma lógica y organización de esta entrevista, se utilizó en las entrevistas a alumnos y comités académicos.

\section{Pauta Entrevista a Docente de Excelencia}

1.- Imagine un docente que se desempeña a nivel de excelencia, ¿Qué competencias debería mostrar? ¿Cuáles de ellas son las más importantes? ¿Por qué las considera más importantes?

2.- Ahora, le pedimos hacer una autoevaluación de usted como docente. ¿Qué competencias presenta usted como docente que lo hace estar bien evaluado por sus alumnos y en su facultad? ¿Cómo se demuestra u observa esa competencia en su docencia?

3.- Respecto a las competencias de docentes destacados, ¿Considera Ud. que deben existir algunas diferencias entre las competencias docentes de los profesores según el nivel en que imparten la docencia (primeros años, en la mitad de la carrera o en los últimos años)? ¿Por qué piensa eso? ¿Puede dar algunos ejemplos?

4.- Sobre competencias básicas del modelo de competencias docentes (se le presentaba a los profesores la dimensión de competencias básicas, con la definición de cada competencia asociada).

a.- Respecto a las siguientes 5 competencias básicas y sus definiciones, ¿Cuál de ellas considera más importantes?, ¿las puede ordenar jerárquicamente?

b.- ¿Cómo se autoevaluaría en estas 5 competencias?

c.- ¿Considera que estas 5 competencias corresponden al perfil de un profesor de excelencia?

(Luego, se le pedía revisar cada competencia básica y pensar en cómo un profesor de excelencia podría dar cuenta de esa competencia, indicando conductas y actividades concretas, cómo esa competencia ayudaba al estudiante en su proceso de aprendizaje y qué dificultades podrían tener para dar cuenta de esta competencia).

5.- Sobre competencias específicas del modelo de competencias docentes (se le presentaba a los profesores la dimensión de competencias específicas, con la definición de cada competencia asociada).

a.- Respecto a las siguientes 3 competencias específicas y sus definiciones, ¿Cuál de ellas considera más importantes?, ¿las puede ordenar jerárquicamente?

b.- ¿Cómo se autoevaluaría en estas 3 competencias?

c.- ¿Considera que estas 3 competencias corresponden al perfil de un profesor de excelencia?

(Luego, se le pedía revisar cada competencia específica y seguir la instrucción de pregunta anterior).

6.- Sobre competencias transversales del modelo de competencias docentes (se le presentaba a los profesores la dimensión de competencias transversales, con la definición de cada competencia asociada).

a.- Respecto a las siguientes 2 competencias transversales y sus definiciones, ¿Cuál de ellas considera más importantes?, ¿las puede ordenar jerárquicamente?

b.- ¿Cómo se autoevaluaría en estas 2 competencias?

c.- ¿Considera que estas 3 competencias corresponden al perfil de un profesor de excelencia?

(Luego, se le pedía revisar cada competencia transversal y seguir la instrucción de la pregunta anterior).

7.- A partir de las 3 dimensiones de revisadas, y las competencias asociadas a cada una de ellas, ¿Cómo sería, para Usted, un perfil docente de excelencia? ¿Sería distinto para profesores que imparten la docencia en distintos niveles? ¿Considera que falta destacar, agregar, re-definir o eliminar alguna competencia mencionada?

En el caso de las observaciones, 2 ayudantes de investigación, egresados de la carrera de psicología, observaron cada clase. Los estudiantes fueron capacitados para cumplir esta labor (tanto en el modelo de competencias docentes como en el uso de la pauta), y realizaron, previamente, prácticas en 5 observaciones de clases, donde recibieron feedback del trabajo realizado, antes de llevar a cabo las observaciones de los docentes de excelencia. Uno de los observadores tenía la instrucción anotar en una ficha cada una de las actividades instruccionales (p.e. exponer contenidos, trabajos en grupo, ejercitación, revisión ejercicios) o no instruccionales (p. e. pasar la lista, mediar un conflicto, interrupciones de clases, 
entrega guías) que realiza el profesor(a), indicando la hora de comienzo y de término. El segundo debía completar una pauta de observación, marcando la frecuencia de la aparición de los indicadores observados (1= bajo, 2 moderado, 3 =alto). A modo de ejemplo, se presentan dos de las dimensiones medidas en la pauta de observación utilizada.

\begin{tabular}{|c|c|c|c|}
\hline Dimensión de Competencias Básicas & 1 & 2 & 3 \\
\hline El profesor/a domina los contenidos que enseña. & & & \\
\hline Las explicaciones del profesor/a son comprensibles & & & \\
\hline El docente capaz de explicar, de distintas formas, hasta lograr que los alumnos comprendan & & & \\
\hline El docente es claro y preciso para transmitir de forma oral los contenidos de la clase & & & \\
\hline El profesor muestra un lenguaje formal y técnico cuando realiza las clases. & & & \\
\hline El profesor utiliza TIC durante sus clases. & & & \\
\hline El profesor muestra motivación por la labor docente. & & & \\
\hline El profesor comienza puntualmente su clase y la termina a la hora estipulada & & & \\
\hline Dimensión de Competencias Transversales (Manejo del Ambiente) & 1 & 2 & 3 \\
\hline El profesor refuerza a los alumnos & & & \\
\hline El profesor se dirige a todos sus alumnos & & & \\
\hline El profesor se preocupa de responder cada duda que tengan los alumnos & & & \\
\hline El profesor busca establecer un diálogo con sus alumnos & & & \\
\hline El profesor mantiene un ánimo positivo durante la clase & & & \\
\hline El ambiente de la clase favorece el aprendizaje (es seguro, positivo, colaborativo) & & & \\
\hline El docente tiene buena disposición para entablar una relación constructiva con los alumnos. & & & \\
\hline La interacción entre profesor y alumno es positiva & & & \\
\hline La interacción entre los alumnos es colaborativa & & & \\
\hline
\end{tabular}

Fig. 2: Dos de las dimensiones medidas en la pauta de observación utilizada

\section{Procedimiento}

En cada disciplina, se le solicitó al director de carrera que, junto al comité académico, seleccionaran a 3 profesores que según su apreciación fueran docentes de "excelencia". Para la selección, utilizaron el criterio de evaluación docente que realizan los estudiantes y de criterios de la facultad. En la universidad, la evaluación docente varía entre 0 y 100, siendo el promedio de 83 puntos aproximadamente. La categoría de excelencia es sobre 91 puntos. Todos los profesores seleccionados, tenían una evaluación docente sobre 95 puntos. Los criterios de Facultad, en general, se referían al compromiso del profesor con su docencia, su participación en reuniones e instancias de capacitación, el cumplimiento de las normativas y el desarrollo de sus cursos.

Se contactó a los profesores sugeridos y se les invitó al estudio, explicándoles en qué consistía. Los docentes que aceptaron participar firmaron un consentimiento. Los profesores fueron entrevistados a través de una pauta semi-estructurada. Cada entrevista fue grabada en audio y tuvo una duración de 90 minutos. Adicionalmente, cada profesor fue encuestado y observado en una de sus clases (que considera dos módulos) utilizando una pauta de observación (la observación la realizaron 2 personas en cada clase). Cada módulo de clases observado tuvo una duración de 80 minutos. El procedimiento de recolección de datos también incorporó la realización de focus group con alumnos de cada carrera (6 estudiantes representativos de los distintos año de la carrera: 1 de primer año, 1 de segundo año, 1 de tercer año, 1 de cuarto año y 2 de quinto año) que tuvieron una duración de 90 minutos. La selección de los estudiantes estuvo a cargo de cada carrera, respetando criterios de asignación equivalente según: año que cursaba la carrera, género y desempeño académico de ellos. Participaron en los focus group un total de 48 estudiantes.

El proceso de recolección y análisis de datos cumplió con los criterios de rigor científico de credibilidad, dependencia, confirmabilidad y transferibilidad (Flick, 2002, LeCompte, 1995; Lincoln y Guba, 1989). La credibilidad fue resguardada al transcribir, de manera literal, las entrevistas y focus group, devolviendo estas transcripciones a los participantes para su revisión y aprobación, para que realmente representaran lo que ellos habían querido expresar. Es posible garantizar la dependencia del estudio debido a que se utilizaron diversas técnicas de recolección de datos (observación, entrevista, focus group) y se enfocó a estudiar el fenómeno desde distintas perspectivas (profesor de excelencia, alumnos, equipos académicos), lo que 
ayuda a entregar estabilidad al estudio y sus conclusiones. La confirmabilidad o auditabilidad tiene relación con el registro y seguimiento de las decisiones tomadas en la investigación, lo que se cuidó grabando en audio las entrevistas y focus group realizados, analizándose transcripciones literales de ellas. Además, el estudio describe adecuadamente el proceso de selección de la muestra, y se contó con una voz externa, de un investigador que no participó del estudio, que colaboró revisando el conjunto de los datos recolectados y enumerando posibles conclusiones, las que eran altamente congruentes con las que habían redactado las autoras del artículo. Finalmente, el criterio de transferibilidad es considerando como cumplido al entregar información respecto a la selección de la muestra y proceso de recolección de datos, mostrándose ejemplos de las pautas de entrevista, el modelo teórico de base y la pregunta de investigación, lo que permite replicarlo.

\section{Análisis de Datos}

En el caso del análisis de los datos cuantitativos, recabados a partir de la pauta de observación y la encuesta a docentes de excelencia, se realizó a través de estadística descriptiva. El análisis consideró una fase preliminar de revisión y limpieza de datos, que se inició con la revisión de la doble digitación, la revisión de los valores atípicos mediante estadísticos multivariados e inspección manual. Los resultados en este nivel se realizaron con estadística descriptiva (medias, desviación estándar, porcentajes, puntajes máximos y mínimos) e inferencial (T-test para comparar medias). En el caso del análisis de los datos cualitativos, recabados a partir de las entrevistas y focus group, éste se realizó a través de análisis de contenido cualitativo. Se realizó codificación abierta y axial, identificándose los temas y definiendo categorías de análisis. En general, las categorías representaban las dimensiones del modelo de competencias docentes y las competencias asociadas. Luego se realizó la integración de información, enriqueciendo las categorías con los datos en cada entrevista.

Finalmente, se realizó una matriz de integración con las categorías analizadas (como filas) y la información proveniente de las entrevistas al docente de excelencia, equipos de gestión académica, alumnos, encuesta profesor y observación profesor (como columnas). Se analizaron los siguientes temas: evidencias del proceso de enseñanza-aprendizaje que dan cuenta de la competencia, nivel de desarrollo de la dimensión y cada competencia asociada, jerarquías de competencias al interior de cada dimensión, diferencias según nivel en que se imparte docencia (cursos de los primeros años, en la mitad, al final de la carrera).

\section{RESULTADOS}

Para claridad de la presentación y discusión de los resultados, esta sección se presenta en cinco subsecciones: Resultados a nivel de observaciones en el aula, Resultados a nivel de entrevistas a los docentes, Resultados a nivel de encuesta a los docentes, Resultados entrevista equipos de gestión académica y Resultados de focus group a estudiantes. Se presentan los resultados más relevantes en cada caso, que contribuyen al objetivo del estudio y no todos los obtenidos.

\section{Resultados a nivel de observaciones en el aula}

Las observaciones realizadas muestran que los docentes presentan alto desempeño en los tres niveles de competencia estudiadas. El promedio de las en las específicas se presentan superan los 2.6 puntos, siendo el máximo 3. El nivel de desempeño más bajo se presenta en las Competencias Específicas, siendo estas diferencias estadísticamente significativas $(\mathrm{t}=2.13, \mathrm{p} \leq .05)$ al comprarlas con las otras dimensiones. La Tabla 2 muestra los resultados en cada uno de los niveles analizados.

Tabla 2: Niveles de las Competencias Docentes a nivel de Dimensiones

\begin{tabular}{|l|c|c|c|c|}
\hline Dimensiones & Promedio & Desviación Típica & Mínimo & Máximo \\
\hline Básicas & 2,74 &, 174 & 2,20 & 3,00 \\
\hline Especificas & 2,63 &, 460 & 1,25 & 3,00 \\
\hline Transversales & 2,73 &, 317 & 2,00 & 3,00 \\
\hline
\end{tabular}

A nivel cualitativo, las principales fortalezas observadas se relacionaron con a) el manejo profundo de los contenidos, el cual se evidenciaba en la claridad de las explicaciones entregadas a los estudiantes; b) el uso de variadas estrategias de enseñanza desplegadas en la clase como exposición, interacción a través de preguntas y comentarios, uso de ejemplos, y relación de los contenidos con la vida profesional; c) la mantención de un clima positivo dentro del aula, mostrando respeto por los alumnos; d) el desarrollo de las clases fue dinámico e interactivo; e) buen manejo del espacio y la voz, manteniendo la atención de los estudiantes; f) buen manejo de grupos y de la disciplina, y finalmente; g) se percibía una vocación real de 
los docentes por el ejercicio de la docencia. Respecto a las áreas de mejora, éstas se pueden orientar en dos ámbitos: a) potenciar una organización y estructura de la clase más clara (inicio, desarrollo y cierre) y b) promover interacciones más desafiantes cognitivamente para los alumnos.

Al analizar las correlaciones entre estos niveles de competencias, se observa que las competencias generales presentan una correlación positiva y significativa con las competencias específicas $(r=.520)$. No se presentaron diferencias significativas para cada uno de los niveles de competencias docentes al considerar el ciclo de formación del alumnado: bachillerato, licenciatura y título.

\section{Resultados a nivel de entrevistas a los docentes}

Las entrevistas realizadas fueron de tipo semi-estructurada, indagando en las competencias que caracterizan al docente universitario de excelencia, analizando como el discurso de los entrevistados se relacionaba con las tres grandes competencias del modelo propuesto. La mayoría de los docentes plantearon que, los docentes destacados muestran conocimiento profundo y actualizado de los contenidos que enseñan. Estos contenidos teóricos se complementan con la experiencia en el área. En este contexto, las experiencias profesionales variadas y la vinculación de los docentes con el mundo laboral, les permite actualizar sus conocimientos, otorgarle significado práctico a los contenidos que enseña y vincular los principios de la teoría con las experiencias y ejemplos que emergen en la práctica profesional.

Los entrevistados enfatizaron la importancia de las competencias comunicativas del docente. En su discurso se pudo identificar dos tipos de habilidades necesarias: las orientadas a la recepción de los mensajes emitidos por los estudiantes, y las orientadas a la emisión de los mensajes o contenidos que al docente le interesa transmitir. En cuanto a las competencias vinculadas a la recepción, la gran mayoría de los docentes destaco la habilidad de escuchar como una competencia clave que debe manejarse en el aula. El docente debiese ser capaz de atender y valorar las opiniones de sus estudiantes, y debe ser sensible a la comunicación del otro, siendo capaz de leer adecuadamente menajes verbales y no verbales que expresan los estudiantes, adaptando sus estrategias de enseñanza a éstos. En cuanto a las habilidades comunicativas orientadas a la emisión, los participantes mencionaron que deben motivar con su lenguaje a la audiencia, de modo de ser persuasivos y expresarse de manera comprensible. Al mismo tiempo, deben mostrar una expresión fluida, transmitiendo los contenidos con claridad y coherencia, manejando los ritmos y tonalidades de la voz a fin de mantener la atención de los estudiantes, así como entregar respuestas claras y precisas ante las preguntas de éstos. Algunos ejemplos de lo planteado por los decentes en este ámbito son:

"[El docente debe ser capaz de] seducir al alumnado, [con] la forma en que hace las clases, la estructura, cómo habla, el buen uso del lenguaje: modular bien, no usar modismos, no usa malas palabras o no adecuadas, ni permitir que ellos lo hagan, exigir a ellos, por ejemplo, buena ortografía” (Entrevista Docente 6).

"El profesor debe ser persuasivo, los alumnos deben creer en él, lo escucha, le creen confían en él" (Entrevista Docente 19).

Las competencias tecnológicas y sociales aparecen escasamente en el discurso de los docentes entrevistados. Estos comprenden el uso de recursos tecnológicos como herramientas complementarias para conectar y motivar a los estudiantes, pero no como algo esencial. Por el contrario, las competencias personales de los docentes son unas de las dimensiones que se nombran con más frecuencia. Los docentes enfatizan una serie de características personales que consideran relevantes. Éstas se han agrupado con fines descriptivos en aquellas orientadas: a) a la relación con los estudiantes, b) a la enseñanza, c) al desarrollo del curso, d) al desarrollo personal. Algunos ejemplos de lo planteado por los decentes en este ámbito son:

"[El docente es capaz de] poner en el lugar del otro (lo que no significa ser menos exigente), ser justo con todos, para que pueda sacar lo mejor de cada uno de ellos. [El docente] entiende que [los estudiantes] pueden tener problemas y les ayuda a solucionarlos (lo que no significa que se los solucione directamente, ni les suba la nota, ni les corra la prueba)" (Entrevista Docente 18).

"[El docente] debe ser éticamente intachable, generar credibilidad entre lo que expresa y lo que vive. Si llega tarde no puede hablar de honradez" (Entrevista Docente 25).

"[El docente debe estar] dispuesto al cambio, buscando estrategias distintas. Nunca hace el curso igual. [El docente] no usa las mismas diapositivas ni realiza el mismo curso todos los años (aunque si el anterior estuvo bueno, no significa que deba cambiar toda la estructura). [Es necesario] que cambie los focos, las asociaciones, que desarrolle salidas a terreno con los estudiantes, que nunca haga el curso exactamente igual, sino que va introduciendo cambios, que son de interés para los estudiantes, que le permiten adaptarse a éstos" (Entrevista Docente 5). 
En la mayoría de los relatos, la cercanía y/o empatía fueron destacadas como rasgos fundamentales del docente. Desde la visión de los entrevistados, deben establecer una relación cercana con los estudiantes, que les permita a estos últimos tener la confianza para plantear sus inquietudes, exponer sus opiniones o acudir al profesor cuando requiere de su ayuda. Adicionalmente, los docentes deben ser capaces de ponerse en el lugar de los estudiantes, sintonizando con sus necesidades, dificultades o motivaciones. El docente de excelencia tiene la habilidad de comprometerse con los estudiantes para que estos logren los aprendizajes esperados en el curso, y es capaz de guiarlos dentro y fuera del horario de clases. Al mismo tiempo, se valora que el docente esté disponible para atender a los estudiantes, y que tenga tiempo para responder las inquietudes de los estudiantes presencialmente o a través de correo electrónico.

Por último, los entrevistados indicaron que las relaciones con los estudiantes se ven favorecidas por la conducta ética del docente. Los docentes deben seguir principios éticos relacionados a calidad, justicia, respeto mutuo, honestidad, e integración de la diversidad, así como demostrar un comportamiento coherente y consistente a través del tiempo con estos valores. Adicionalmente, la motivación del docente por la enseñanza fue frecuentemente enfatizado, lo que les permite involucrarse en el proceso, invertir tiempo y esfuerzo en sus estudiantes, y transmitir a estos la pasión por el área o disciplina particular que enseñan. Para profundizar en las competencias específicas, se buscó indagar en otras competencias pedagógicas que los docentes consideraran relevantes de destacar en un profesor de excelencia. Con la finalidad de organizar los hallazgos en estas dimensiones, éstos se presentarán en tres grupos: a) planificación, b) enseñanza y c) evaluación.

Los participantes de la investigación varían en sus percepciones acerca de la utilidad y relevancia de las habilidades de planificación y organización. Estas habilidades se ven reflejadas en la capacidad del docente de crear un cronograma de actividades para el curso, así como de seguir habitualmente los contenidos y plazos indicados en el programa del curso y la calendarización. Por otro lado, las habilidades de planificación y organización se reflejan en que el docente llega a la clase con la sesión preparada, considerando tanto el detalle de las actividades como los recursos que serán utilizados. Además, el profesor presenta al inicio de cada clase los objetivos de la sesión y los vincula tanto con los contenidos revisados con anterioridad, como con aquellos que serán revisados en el futuro.

Al respecto uno de los consultados señala: "[El docente posee una] planificación previa, sabe cuál es el tema que va a tratar en cada clase, sabe introducirlo, recordando lo que han visto en la clase pasada. [El docente] da a conocer los objetivos de clase, generando una articulación con lo visto antes y con lo que viene después" (Entrevista Docente 1).

Los profesores que perciben utilidad en ésta, señalan que a los alumnos la calendarización les permite organizarse y manejar su tiempo, favoreciendo la autorregulación del proceso de aprendizaje. A partir de ésta pueden planificar tiempos de estudio, preparación de actividades y evaluaciones, así como administrar sus inasistencias a clase y traer a la misma los materiales necesarios. Por otro lado, la explicitación de los objetivos de clase como estrategia, les permite comprender el significado de cada actividad para su aprendizaje y la vinculación entre distintos contenidos a lo largo del curso, lo que favorece la motivación y el logro de las competencias esperadas.

En concordancia uno de los docentes ha expresado: "La mayor parte de los alumnos valoran la calendarización, aunque algunos no la toman en cuenta, no son conscientes de ella, pero ellos sienten cuando el curso está planificado. [Es útil] porque los alumnos necesitan estructura, organizar sus tiempos, saber de las evaluaciones, anticipar lecturas" (Entrevista Docente 5).

El segundo grupo de habilidades se vinculó con la capacidad de implementar estrategias o condiciones que permitan mejorar la transmisión del conocimiento y desarrollar las competencias del curso en los estudiantes. Los entrevistados destacan el manejo de variadas estrategias de aprendizaje, como una herramienta para alcanzar los objetivos del curso. La mayoría de ellos refiere a la capacidad de motivar a sus estudiantes a participar activamente en las actividades de la clase y a comprometerse con los objetivos del curso. Igualmente, algunos docentes expresan que el proceso de enseñanza y aprendizaje se ve favorecido si el profesor es capaz de ser exigente con los estudiantes. El docente debe ser capaz de desafiar los límites de los estudiantes, para que estos puedan aprovechar su potencial, confiando en sus capacidades.

En cuanto a evaluación, los docentes reportan una serie de dificultades en su implementación, aunque destaca que diferentes docentes aluden a dificultades de distinto tipo. Algunas de las limitaciones de este proceso se encuentran relacionadas con la construcción de los instrumentos de evaluación. En este sentido, los docentes manifiestan que poseen dificultades para construir ítems de evaluación que sean suficientemente desafiantes y novedosos, así como de construir instrumentos de evaluación acorde a los objetivos del curso y que permitan 
realmente reflejar los logros de aprendizaje de los alumnos a lo largo del proceso. Al mismo tiempo las evaluaciones desarrolladas suelen ser demasiado extensas, lo que genera molestia en los estudiantes. Por otro lado, los docentes muestran dificultades para innovar en las formas de evaluación, ya que carecen de tiempo para su preparación y corrección y desconocen otras estrategias de evaluación distintas a las aplicadas tradicionalmente. Los profesores tienen dificultades para desarrollar evaluaciones durante el proceso de enseñanza y aprendizaje, así como para realizar evaluaciones no calificadas e implementar evaluaciones personalizadas, que atiendan a las características particulares de los estudiantes.

Respecto a la tercera dimensión del modelo, competencias transversales, los docentes destacaron las habilidades relacionadas a promover un clima adecuado en el aula. En este contexto, se destaca el liderazgo en el aula, que les permite manejar de forma eficiente las dinámicas del curso. El docente debe tener energía que le permita manejar el comportamiento de los estudiantes durante clases, estimulando la atención sostenida, el silencio, el respeto mutuo y la adecuada solución de los problemas que surgen en el marco de la interacción del aula. Finalmente, el manejo del clima del aula se verá favorecido, según los consultados, por la flexibilidad del docente para adaptar las estrategias y las actividades inicialmente planificadas, a las dinámicas particulares de cada clase, a los resultados académicos de los estudiantes, así como los intereses y prioridades planteadas por los mismos estudiantes. En este sentido, el docente debe tener, en todo momento claridad de los objetivos y el avance en la consecución de las actividades planeadas del curso, para priorizar acciones que contribuyan a alcanzar los mismos y abordar todos los temas predefinidos.

Los docentes reportan problemas con la competencia de reflexión-investigación ya que señalan que se les dificulta analizar críticamente las estrategias implementadas durante el desarrollo del curso, o contar con una visión externa de esto que les permitan aprender y mejorar sus prácticas. Una de las personas consultadas señala: "[Como docentes nos] falta autocrítica y autoevaluación de las estrategias utilidades. Nos cuenta pensar en qué nos equivocamos, en algún punto del proceso. No hacemos esta evaluación en el momento adecuado (por ejemplo, durante el curso y no luego de éste), porque nos falta tiempo y motivación para realizar esta reflexión académica, que nos permita saber cómo mejorar las prácticas" (Entrevista Docente 8).

\section{Resultados a nivel de encuesta a los docentes}

Con el objetivo de conocer la importancia que los docentes les dan a las habilidades presentadas en cada dimensión del modelo propuesto, se les solicitó ordenarlas acorde a su relevancia, a partir de una encuesta. Los resultados se presentan en la Tabla 3, mostrando el porcentaje asignado desde el primer al quinto lugar de relevancia. La Figura 3 muestra las competencias indagadas en orden de relevancia.

Tabla 3: Relevancia dada por los docentes a las competencias básicas

\begin{tabular}{|c|c|c|c|c|c|c|}
\hline Ordenamiento & & Cognitivas & Sociales & Comunicativas & Tecnológicas & Personales \\
\hline Primer lugar & & $70 \%$ & 0 & $15 \%$ & $8 \%$ & $19 \%$ \\
\hline Segundo lugar & & $27 \%$ & $8 \%$ & $47 \%$ & $23 \%$ & $19 \%$ \\
\hline Tercer lugar & & - & $23 \%$ & $23 \%$ & - & $46 \%$ \\
\hline Cuarto lugar & & $3 \%$ & $43 \%$ & $15 \%$ & - & $12 \%$ \\
\hline Quinto lugar & & - & $26 \%$ & - & $69 \%$ & $4 \%$ \\
\hline Cognitivas & $\Rightarrow$ & Comunicativas & $\Rightarrow$ & onales & Sociales & Tecnológicas \\
\hline
\end{tabular}

Fig 3: Competencias básicas docentes en orden relevancia según percepción de los docentes

Respecto de las competencias específicas, los docentes también jerarquizaron las habilidades correspondientes a este grupo. En la Tabla 4 se presenta el porcentaje con el que los docentes asignaron cada competencia específica desde el primer hasta el quinto lugar de relevancia. En consideración a estos resultados, la Figura 4 presenta las habilidades estudiadas en orden de relevancia. En general, los docentes se evalúan con un alto desempeño en todas las habilidades. Las habilidades de planificación y organización corresponden a la dimensión en la que los docentes se evaluaron con un mejor desempeño, alcanzando un $88 \%$ de logro. En contraste, el desempeño más bajo es en las habilidades de reflexión e investigación con un $76 \%$ de logro. 
Tabla 4: Relevancia asignada por los docentes a las competencias específicas

\begin{tabular}{|l|c|c|c|c|c|}
\hline Ordenamiento & $\begin{array}{c}\text { Planificación } y \\
\text { organización }\end{array}$ & Didácticas & Evaluación & \multicolumn{1}{c}{$\begin{array}{c}\text { Manejo del } \\
\text { clima }\end{array}$} & $\begin{array}{c}\text { Reflexión e } \\
\text { investigación }\end{array}$ \\
\hline Primer lugar & $38 \%$ & $35 \%$ & $4 \%$ & $12 \%$ & $19 \%$ \\
\hline Segundo lugar & $23 \%$ & $38 \%$ & $15 \%$ & $35 \%$ & - \\
\hline Tercer lugar & $23 \%$ & $15 \%$ & $20 \%$ & $26 \%$ & $12 \%$ \\
\hline Cuarto lugar & $4 \%$ & $12 \%$ & $38 \%$ & $15 \%$ & $27 \%$ \\
\hline Quinto lugar & $12 \%$ & - & $23 \%$ & $12 \%$ & $42 \%$ \\
\hline
\end{tabular}

Planificación y organización
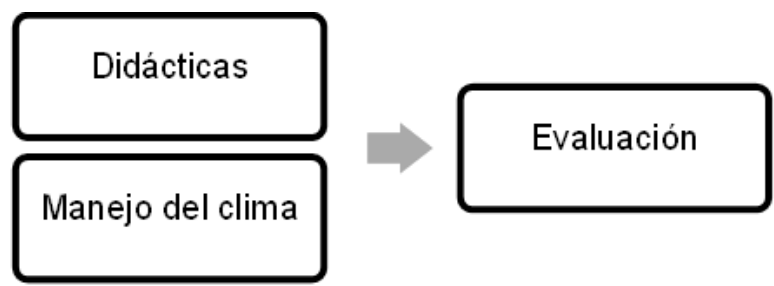

Reflexión e investigación

Fig. 4: Competencias vinculadas a la enseñanza y aprendizaje en orden relevancia.

\section{Resultados entrevista equipos de gestión académica}

A continuación, se presentan los resultados de este trabajo, en las siguientes secciones: (i) Competencias Básicas; (ii) Competencias Específicas; (iii) Competencias Transversales; (iv) Resultados de la entrevista grupal a estudiantes; (v) Descripción de Niveles de Competencias Docentes: Competencias Básicas; (vi) Descripción de Niveles de Competencias Específicas Docentes y; (vii) Descripción de Niveles de Transversales Docentes; (viii) Jerarquía otorgada por los alumnos

\section{Competencias Básicas}

Se solicitó a los equipos directivos ordenar las competencias docentes generales, en función de su relevancia. Los resultados obtenidos fueron los mostrados en la Tabla 5.

Tabla 5: Relevancia asignada por los equipos docentes a las competencias básicas

\begin{tabular}{|c|c|c|c|c|c|}
\hline Ordenamiento & Cognitivas & Sociales & Comunicativas & Tecnológicas & Personales \\
\hline Primer lugar & $70 \%$ & & $10 \%$ & & $20 \%$ \\
\hline Segundo lugar & & & $60 \%$ & & $20 \%$ \\
\hline Tercer lugar & $20 \%$ & & $30 \%$ & & $60 \%$ \\
\hline Cuarto lugar & $10 \%$ & $90 \%$ & & $10 \%$ & \\
\hline Quinto lugar & & $10 \%$ & & $90 \%$ & \\
\hline Cognitivas & Comu & tivas & Personales & Sociales & Tecnológicas \\
\hline
\end{tabular}

\section{Competencias Especificas}

Los comités académicos jerarquizaron cada competencia específica. Los resultados fueron los mostrados en la Tabla 6.

Tabla 6: Relevancia asignada por los equipos docentes a las competencias específicas

\begin{tabular}{|l|c|c|c|}
\hline Ordenamiento & $\begin{array}{l}\text { Planificación } \\
\text { organización }\end{array}$ & Didácticas & Evaluación \\
\hline Primer lugar & $20 \%$ & $70 \%$ & $10 \%$ \\
\hline Segundo lugar & $20 \%$ & $20 \%$ & $60 \%$ \\
\hline Tercer lugar & $60 \%$ & $10 \%$ & $30 \%$ \\
\hline Didácticas & Evaluación & Planificación \\
\hline
\end{tabular}




\section{Competencias Transversales}

Los comités académicos jerarquizaron cada competencia transversal. Los resultados fueron los siguientes:

Tabla 7: Relevancia asignada por los equipos docentes a las competencias transversales

\begin{tabular}{|c|c|c|}
\hline Ordenamiento & Manejo del clima & Reflexión e investigación \\
\hline Primer lugar & $80 \%$ & $20 \%$ \\
\hline \multirow[t]{2}{*}{ Segundo lugar } & $20 \%$ & $20 \%$ \\
\hline & $\begin{array}{l}\text { Manejo de } \\
\text { Clima }\end{array}$ & $\begin{array}{l}\text { exión e } \\
\text { stigació }\end{array}$ \\
\hline
\end{tabular}

\section{Resultados de la entrevista grupal a estudiantes}

El objetivo era evaluar las competencias de "un buen docente" desde la perspectiva de los estudiantes. Éstos señalaron distintas características de un docente ideal. Un total de 14 cualidades fueron determinadas, sus nombres y frecuencias se presentan en la Tabla 5.

Tabla 8: Frecuencia de competencias de un docente de excelencia descritas por los estudiantes

\begin{tabular}{|c|c|c|c|}
\hline Cualidad & Descripción & Frecuencia & $\begin{array}{l}\text { Orden de } \\
\text { importancia }\end{array}$ \\
\hline Accesible & Cercano, empático, flexible, humilde, disponible & $23 \%$ & 4 \\
\hline Dinámico & $\begin{array}{l}\text { Interactúa con los alumnos, la clase es participativa, es entretenido, } \\
\text { ocupa distintas estrategias para enseñar. }\end{array}$ & $13 \%$ & 3 \\
\hline Práctico & Lleva la teoría a la práctica, es una clase aplicada, ejemplifica. & $12 \%$ & 5 \\
\hline Actualizado & $\begin{array}{l}\text { Tiene un profundo conocimiento de lo que enseña y sabe de lo nuevo } \\
\text { en el área. }\end{array}$ & $12 \%$ & 1 \\
\hline Responsable & $\begin{array}{l}\text { Cumple con los compromisos en la entrega de notas y trabajos, es } \\
\text { puntual, respetuoso con los alumnos. }\end{array}$ & $9 \%$ & 7 \\
\hline Exigente & $\begin{array}{l}\text { Es estricto en los aspectos formales (no deja usar celular, conversar } \\
\text { o llegar tarde) y exigente en lo académico. Tiene altas expectativas } \\
\text { sobre lo que pueden aprender los alumnos. }\end{array}$ & $7 \%$ & 2 \\
\hline Motivado & Muestra pasión por enseñar. Tiene vocación. Motiva a los alumnos. & $4,5 \%$ & 6 \\
\hline Liderazgo & Es una autoridad, sabe manejar al grupo. & $4,5 \%$ & 8 \\
\hline Sabe comunicar & Es claro, se comunica bien. Adecuada expresión corporal. & $4,5, \%$ & 9 \\
\hline $\begin{array}{l}\text { Destacado en su } \\
\text { carrera }\end{array}$ & $\begin{array}{l}\text { Tiene experiencia laboral, no es recién egresado. Tiene experiencia } \\
\text { en lo que enseña. }\end{array}$ & $4,5 \%$ & 10 \\
\hline Integral & $\begin{array}{l}\text { Sabe de todo no sólo la materia que enseña. Es justo. Es un ejemplo } \\
\text { a seguir. }\end{array}$ & $3 \%$ & \\
\hline Clase organizada & La clase tiene un orden, una estructura. No se va por las ramas. & $1,5 \%$ & \\
\hline Retroalimenta & No sólo entrega las notas, informa lo que estaba bien y mal. Corrige. & $1,5 \%$ & \\
\hline
\end{tabular}

Todos los estudiantes mencionan que la docencia va cambiando a medida en que ellos van avanzando en su carrera. Ellos señalan 7 dimensiones que cambiaron, 5 de estas incrementaron a medida a que progresan en los cursos de la carrera (exigencia, complejidad, flexibilidad, confianza, practica) y dos disminuyen a lo largo de ésta (apoyo, teoría). Para los estudiantes, los docentes que enseñan en primer y segundo año (Nivel de Bachillerato: primer y segundo año), debiesen presentar características como accesibilidad y empatía, motivación, capacidad de enseñar, y que sean exigentes. A continuación, en la Tabla 7 se presenta el listado de características enunciadas para profesores de primer y segundo año y su frecuencia de aparición. Las características que un docente de tercer y cuarto año (Nivel de Licenciatura), debería tener según los estudiantes, se muestran en la Tabla 8. Finalmente, en quinto año (Nivel de Título), los estudiantes describen a docentes con las características deseadas, presentadas en la Tabla 9. 
Tabla 9: Características de los docentes de primer y segundo año (Bachillerato), según los estudiantes

\begin{tabular}{|l|c|}
\hline Características del Profesor de Bachillerato & Frecuencia \\
\hline Motivador & $20 \%$ \\
\hline Accesibilidad, Cercanía, Empatía & $15 \%$ \\
\hline Exigente & $15 \%$ \\
\hline Que tenga la capacidad de enseñar & $10 \%$ \\
\hline Que realice salidas a terreno & $10 \%$ \\
\hline Que aplique la teoría en ejemplos prácticos & $10 \%$ \\
\hline Que retroalimente & $10 \%$ \\
\hline Paciente & $5 \%$ \\
\hline Que tenga vocación & $5 \%$ \\
\hline
\end{tabular}

Tabla 10: Características de los docentes de tercer y cuarto año (Licenciatura), según los estudiantes

\begin{tabular}{|l|c|}
\hline Características del Profesor de Licenciatura & Frecuencia \\
\hline $\begin{array}{l}\text { Que tengan experiencia laboral en el área y sea } \\
\text { expertos en el tema }\end{array}$ & $17 \%$ \\
\hline $\begin{array}{l}\text { Compromiso con la enseñanza y el aprendizaje de } \\
\text { los alumnos }\end{array}$ & $17 \%$ \\
\hline Que muestren disposición y cercanía & $17 \%$ \\
\hline Que preparen sus clases & $17 \%$ \\
\hline Que aplique la teoría en ejemplos prácticos & $17 \%$ \\
\hline Que ayude a los alumnos a "integrar" contenidos & $7,5 \%$ \\
\hline Que se estricto y exigente & $7,5 \%$ \\
\hline
\end{tabular}

Tabla 11: Características de los docentes de quinto año (Título), según los estudiantes

\begin{tabular}{|l|c|}
\hline Características del Profesor de Título & Frecuencia \\
\hline Muestran Cercanía, Accesibilidad y Empatía & $25 \%$ \\
\hline Entregan Apoyo, Consejo y Guía & $25 \%$ \\
\hline Entregan Herramientas Laborales & $10 \%$ \\
\hline Son exigentes & $10 \%$ \\
\hline Retroalimentan & $10 \%$ \\
\hline Entregan libertad y facilidad para la creatividad & $10 \%$ \\
\hline Actualizados & $5 \%$ \\
\hline Investigan & $5 \%$ \\
\hline
\end{tabular}

\section{Descripción de Niveles de Competencias Docentes: Competencias Básicas.}

Los estudiantes analizaron las competencias generales de un docente, que corresponden a las: cognitivas, sociales, comunicativas, tecnológicas y personales, indicando el orden de importancia que tienen en un profesor. El orden más frecuentemente entregado es el mostrado en la Tabla 12.

Tabla 12: Relevancia asignada por los alumnos a las competencias básicas

\begin{tabular}{|c|l|}
\hline Orden & Competencias Básicas \\
\hline 1 & Cognitivas \\
\hline 2 & Comunicativas \\
\hline 3 & Personales \\
\hline 4 & Sociales \\
\hline 5 & Tecnológicas \\
\hline
\end{tabular}

Los alumnos consideran que las menos presentes en sus docentes son las competencias: sociales (capacidad de colaboración y trabajo en equipo que muestra el docente en la asignatura que imparte, con el resto de profesores, la facultad y universidad) y tecnológicas (incorporación y dominio de tecnologías de comunicación e información (TICs) para potenciar el aprendizaje). Pero consideran que ésta última no es tan relevante. 


\section{Descripción de Niveles de Competencias Específicas Docentes}

Los estudiantes analizaron las competencias generales de un docente, que corresponden a las: cognitivas, sociales, comunicativas, tecnológicas y personales, indicando el orden de importancia que tienen en un profesor. El orden más frecuentemente entregado en la Tabla 13. A los estudiantes se les pidió describir cuáles serían las evidencias de estas competencias específicas. El resultado es mostrado en la Tabla 14.

Tabla 13: Relevancia asignada por los alumnos a las competencias específicas

\begin{tabular}{|c|l|}
\hline Orden & Competencias \\
\hline 1 & Habilidades Didácticas \\
\hline 2 & Habilidades de Planificación \\
\hline 3 & Evaluación \\
\hline
\end{tabular}

Tabla 14: Evidencias de las competencias específicas

\begin{tabular}{|l|l|l|}
\hline \multicolumn{1}{|c|}{ Planificación } & \multicolumn{1}{|c|}{ Didáctica } & \multicolumn{1}{c|}{ Evaluación } \\
\hline & -Los alumnos participan en clases. El & -Test semanal. \\
-Clase organizada & profesor interactúa con los & - Guías obligatorias. \\
-Se distribuyen adecuadamente los & -Se relaciona la teoría con la práctica. & -Certamen Acumulativo. \\
tiempos de las clases en relación a & -El profesor da ejemplos. & -Realiza preguntas en clases. \\
los contenidos del programa. & -El profesor motiva. & -Corrige lo bueno y lo malo. \\
-Están calendarizadas las fechas de & -Vemos películas, videos. & -Trabaja con las equivocaciones de \\
las evaluaciones y actividades & nosotros. \\
académicas. & -Se realiza trabajo grupal. & -Se preocupa del proceso y no sólo \\
-Se comprenden los objetivos de la & -Se relacionan los contenidos con la & del resultado. \\
asignatura. & vida laboral. & -Aplica la materia en las \\
-Existe una relación entre la clase & -Se invitan otros docentes o expertos & evaluaciones. \\
anterior y la siguiente. & a algunas clases. & -Retroalimenta oralmente. \\
-Se mantiene un ritmo en las clases. & -El PowerPoint es preciso, no tiene & -Sanciona cuando no se cumplen \\
-Se cumplen los plazos. & exceso de palabras. & las reglas. \\
& -El profesor comparte su propia & -Solicita presentaciones \\
individuales. \\
experiencia. & -Realiza exámenes orales. \\
& -Se realizan salidas a terreno. & -Evalúa actividades prácticas. \\
\hline
\end{tabular}

\section{Descripción de Niveles de Transversales Docentes}

Los estudiantes analizaron las competencias transversales, indicando el orden de importancia que tienen en un profesor. El orden más frecuentemente entregado es el siguiente:

Tabla 15: Relevancia asignada por los alumnos a las competencias Transversales

\begin{tabular}{|r|l|}
\hline Orden & Competencias \\
\hline 1 & Manejo del Ambiente \\
\hline 2 & Reflexión-Investigación \\
\hline
\end{tabular}

Los alumnos consideran que las menos presentes en sus docentes son las competencias: ReflexiónInvestigación (capacidad del profesor de investigar y reflexionar sobre su práctica docente y su efectividad, probando cambios en metodologías de enseñanza y evaluación en pro de mejores resultados).

\section{Jerarquía otorgada por los alumnos}

Los estudiantes ordenaron, desde mayor a menor importancia, las habilidades asociadas a los tres niveles de competencias docentes analizadas, llegando al siguiente listado: 
Tabla 16: Jerarquía entregada por los alumnos sobre las 10 competencias del modelo.

\begin{tabular}{|c|l|}
\hline Orden & Competencias \\
\hline 1 & Cognitivas \\
\hline 2 & Didácticas \\
\hline 3 & Comunicativas \\
\hline \multirow{2}{*}{4} & Planificación \\
\cline { 2 - 2 } & Personales \\
\hline 5 & Manejo del Ambiente \\
\hline \multirow{2}{*}{6} & Evaluación \\
\cline { 2 - 2 } & Reflexión-Investigación \\
\hline 7 & Tecnológicas \\
\hline 8 & Sociales \\
\hline
\end{tabular}

\section{DISCUSIÓN}

Existe una tensión constante en la mayoría de las universidades en el mundo respecto a qué es más importante dentro de la carrera docente: la docencia o la investigación. Muchos estarán de acuerdo en que la docencia es más valorada en el discurso de las autoridades, pero la investigación resulta reconocida en términos reales como un área de ascenso en las categorías docentes establecidas por cada universidad. Esta división es perjudicial para la formación profesional de los estudiantes, porque éstos requieren no solo de docentes expertos y motivados en enseñar sus contenidos, sino que también reflexivos y autocríticos respecto de las metodologías que utilizan, investigando sobre la efectividad de éstas.

Los docentes deben preocuparse no solo de formar en contenidos a los futuros profesionales, sino que también invertir en esfuerzos por lograr perfiles de egreso de alta complejidad, que incluyen el desarrollo de procedimientos, habilidades de pensamiento de orden superior, y actitudes. Esto convierte a la docencia en una actividad extremadamente desafiante, ya que, no sólo implica el traspaso de conocimiento, sino que conlleva un alto grado de responsabilidad, puesto que es el docente quién debe planificar y tomar importantes decisiones, cómo qué contenidos priorizar, qué estrategias utilizar en la enseñanza, como evaluar el aprendizaje, cómo tratar con los estudiantes, entre otros. Estas decisiones tendrán un fuerte impacto en la calidad de la experiencia de aprendizaje de los estudiantes y en cómo éstos se comportan en el mundo laboral, una vez que se conviertan en profesionales. Como se mostró en el apartado introducción del presente artículo, la respuesta a cuál es el perfil de competencias del docente universitario, no es simple, y no parece existir un consenso por parte de la literatura. Existen diversas miradas, que sugieren que cada institución de educación superior debe seleccionar cuál de todos estos modelos se ajusta mejor a su modelo educativo y es útil para seleccionar, capacitar, y evaluar el desempeño de sus docentes considerando un mínimo de competencias requeridas.

Desde la perspectiva de este estudio, existen tres dimensiones que no pueden estar ausentes al momento de pensar en las competencias de un docente universitario: las competencias básicas, las específicas, y las transversales. Las competencias básicas, son consideradas mínimas para el ejercicio de la práctica docente (por ejemplo Cano, 2005; Casero, 2008; Monereo y Domínguez, 2014). Las competencias específicas se refieren a aquellas relacionadas con la capacidad de enseñar con la idea de promover una experiencia profunda y significativa en los estudiantes (planificación, didáctica, evaluación) tal como lo proponen autores como Galvis (2007), Zabalza (2009) y Ortega (2010). Este estudio sugiere que estas son las más importantes y diferenciadoras respecto de quien es un buen docente. Finalmente, las competencias transversales (capacidad de mantener un clima positivo en el aula, reflexionar sobre las prácticas pedagógicas, y mantener interacciones de buena calidad con los estudiantes) otorgan un componente afectivo y están vinculadas a aspectos relacionales (Camargo y Pardo, 2008; Elton, 1996, entre otros). Es así como se aboga por un docente que no sólo enseñe bien, sino que también sea capaz de entender el proceso de enseñanza-aprendizaje como una red compleja, que implica planificar cuidadosamente las acciones, reflexionar acerca de éstas mientras se estén llevando a cabo, evaluar el aprendizaje de los estudiantes y relacionarse bien con éstos.

En cuanto a los resultados de esta investigación, las observaciones en aula permiten concluir que los docentes evidencian altos desempeños en todas las competencias propuestas por el modelo. En general, dominan los contenidos y se adecuan a los estudiantes a quienes enseñan, preocupándose, por estructurar la clase y motivando a los estudiantes a establecer relaciones entre éstos, en un clima positivo. Sin embargo, los docentes muestran dificultades para innovar y utilizar las TICs, explicar los objetivos de la clase, realizar un cierre, retroalimentar a los estudiantes e incentivarlos a encontrar las respuestas a sus 
preguntas. Esto se suma a que existen pocas instancias de diálogo y que la estrategia más utilizada es la clase expositiva con apoyo de PowerPoint. Esto permite concluir, que en términos de las prácticas que se llevan a cabo en las clases, los docentes parecen estar ubicados en un polo tradicional de la docencia, realizando clases frontales, centradas en los contenidos y en la profunda comprensión de éstos. Las estrategias de enseñanza activas y centradas en los estudiantes son escasas. En la mayoría de los casos, los objetivos o resultados del aprendizaje no son explicitados, y parecer no existir una introducción ni conclusión de la clase, así como tampoco se utilizan tecnologías ni innovaciones docentes.

En los resultados de las entrevistas individuales, el discurso de los docentes es consistente con las observaciones. Los docentes valoran en gran medida las competencias cognitivas, comunicativas y pedagógicas. Sin embargo, en su discurso no aluden a competencias sociales y tecnológicas, y al solicitarles que las jerarquicen, éstas son ubicadas al final. Lo que es consistente con que en la práctica docente éstas tampoco se evidencian.

Respecto de la percepción de los estudiantes, abordada a través de la técnica de focus group, los estudiantes, a diferencia de los docentes, valoraron primero las competencias trasversales, esperando que los docentes sean "cercanos, empáticos, flexibles, humildes, y disponibles". Además, que exista un alto grado de interacción, participación y entretención. En segundo lugar, sitúan las competencias básicas y las específicas aparecen en el último lugar. Esto en contraste a lo encontrado por diversos estudios que muestran su importancia en el proceso de aprendizaje, aquí los estudiantes prácticamente no la mencionan (con sólo una frecuencia de 1,5\% en su discurso). También, es interesante que los estudiantes diferencien estas características dependiendo del nivel en el cual el docente realiza clases. De esta forma, se valoran más capacidades de motivación en los primeros años, mientras que se prefiere un rol de guía en quinto año.

Finalmente es posible concluir que este trabajo intenta ser una aproximación exploratoria, al ámbito de las competencias docentes. Su contribución y originalidad recaen en la búsqueda de mostrar una mirada que involucre a todos los actores del proceso de enseñanza-aprendizaje en la educación superior. De esta forma, es posible establecer que se cumplió el objetivo propuesto por este trabajo, ya que por un lado, se presentó un modelo teórico de competencias pedagógicas que define a un docente de excelencia, y por otro se mostró como esté modelo se relaciona con la percepción de docentes, estudiantes y equipos de gestión académica.

\section{CONCLUSIONES}

A partir de los resultados obtenidos, se pueden indicar las siguientes conclusiones: 1) En las observaciones realizadas a los docentes, queda manifiesto que éstos se destacan en las 3 competencias propuestas por el modelo (Básicas, Específicas y Transversales), lo que resulta coherente con el hecho de que se trate de profesores que se destacan en su evaluación docente; 2) Desde la perspectiva de los estudiantes, la competencia más esperada fue la accesibilidad, entendida como la cercanía, empatía, flexibilidad, humildad y disponibilidad, seguida por la actualización del conocimiento. También se observaron diferencias en los estudiantes, según su nivel de estudios. Los estudiantes de bachillerato, valoraron la capacidad de motivar del docente, a diferencia de los de licenciatura, que destacaron la experiencia laboral y experticia en el tema, y los de título que esperan cercanía, accesibilidad y empatía; 3) Las entrevistas llevadas a cabo a los profesores evidencian que las Competencias Básicas, que valoran, son el manejo profundo y actualizado de los contenidos que enseñan, los que se complementan con experiencia en el área, seguidas por las competencias comunicativas y las características personales. A nivel de Competencias Específicas, destacan la capacidad de motivar a los estudiantes y de promover la autonomía, mientras que a nivel de Competencias Transversales, la habilidad para promover un clima adecuada en el aula; y 4) En las encuestas, los docentes consideraron como más relevantes las competencias cognitivas, dentro de las Básicas, y las de planificación y organización, dentro de las Específicas. Estos hallazgos, muestran que los diferentes actores otorgan importancia a diferentes competencias.

\section{REFERENCIAS}

Abadía, A.R., Bueno, C., y otros 5 autores, Competencias del buen docente universitario. Opinión de los estudiantes, Revista de docencia Universitaria, 13(2), 363-390 (2015)

Alvarez-Rojo, V., Romero, S. y otros 13 autores, Necesidades de formación del profesorado universitario para la adaptación de su docencia al Espacio Europeo de Educación Superior (EEES), Relieve, 17(1), 23- 37 (2011)

Baeten, M., Struyven, K. y Dochy, F., Student-centred teaching methods: Can they optimise students' approaches to learning in professional higher education? Studies in Educational Evaluation, 39(1), 14-22. (2013) 
Biggs, J. y Tang, C., Teaching for quality learning at university: What the student does, Maidenhead, Berkshire, Open University Press (2011)

Brunner, J., Medio siglo de transformaciones de la educación superior chilena: un estado del arte. En La educación superior de Chile: Transformación, desarrollo y crisis, por A. Bernasconi, Ediciones U.C., 21-107, Santiago de Chile (2015)

Camargo, I. y Pardo, C., Competencias docentes de profesores de pregrado: Diseño y validación de un instrumento de evaluación, Universitas Psychologica, 7(2), 401- 455 (2008)

Camperos, M., La evaluación por competencias, mitos, peligros y desafíos, Educere, 43, 805- 814 (2007)

Cano, E., Cómo mejorar las competencias de los docentes. Guía para la autoevaluación y el desarrollo de las competencias del profesorado, Graó, Barcelona (2005)

Cardona, A., Barrenechea, M., Mijangos, J.J. y Olascoaga, J., Concepto y determinantes de la calidad en la educación superior. Un sondeo de opinión entre profesores de universidades españolas, Archivos Analíticos de Políticas Educativas, 17(10), 1-25 (2009)

Casero, A., Propuesta de un cuestionario de evaluación de la calidad docente universitaria consensuado entre alumnos y profesor, Revista de Investigación Educativa, 26 (1), 25-44 (2008)

Cid, A., Zabalza, M.A., y Doval, M.A.L. La docencia universitaria: un modelo para su análisis. Revista de Docencia Universitaria, 10(1), 87-104 (2012)

Comellas, M.J., Las competencias del profesorado para la acción tutorial, Praxis, Barcelona (2002)

Çubukçu, Z, Teachers' evaluation of student-centered learning environments, Education, 133 (1), 49-66, (2012)

Devlin, M. y O'Shea, H., Effective university teaching: views of Australian university students from low socioeconomic status backgrounds, Teaching in Higher Education, 17 (4), 385-397 (2012)

Elton, L., Criteria for teaching competence and teaching excellence in higher education. In Aylett, R. and Gregory, K., Evaluating Teacher Quality in Higher Education, The Falmer Press, London (1996)

Feijoo, R. M. A., y Cerro-Ruiz, M.J.B., Perfiles docentes y excelencia: un estudio en la Universidad Técnica Particular de Loja, Ecuador, Revista Iberoamericana de Educación a Distancia, 18(2), 225-250 (2015)

Fernández-Cruz, M. y Gijón, J., Nuevas políticas de profesionalización docente en la Educación Superior, Journal for Educators, Teachers and Trainers, 2, 92-106 (2011)

Flick, U., An Introduction to Qualitative Research, Sage Publications (2002)

Gairín, J., Formación de profesores basada en competencias, Bordón, 63 (1), 93-108 (2011)

Galvis, R., De un perfil docente tradicional a un perfil docente basado en competencia, Acción Pedagógica, $16,48-57(2007)$

Ghedin, E. y Aquario, D., Moving toward multidimensional evaluation of teaching in higher education: a study across faculties, Higher Education, 56, 583-597 (2008)

Gómez-Mendoza, M.A. y Alzate-Piedrahíta, M.V., La alegre entrada y el irresistible ascenso de las competencias en la universidad, Educación y Educadores, 13(3), 453-474 (2010)

González, C., Perspectivas y desafíos de la docencia en la educación superior chilena. En Bernasconi, A. La Educación Superior en Chile. Transformación, desarrollo y crisis, Ediciones U.C., Santiago de Chile (2015)

Hernández-Sampieri, R., Fernández, C. y Baptista, P., Metodología de la Investigación, Mc Graw Hill Education, $6^{\text {ta }}$ Ed., Lima-Perú (2014) 
Juliá, M. T., Formación basada en competencias: Aportes a la calidad de los aprendizajes en la formación de psicólogos. En Catalán, J., Psicología educacional. Proponiendo rumbos, problemáticas y aportaciones, Editorial Universidad de la Serena, La Serena-Chile (2012)

Knight, P., El profesorado de Educación Superior, Formación para la Excelencia, Narcea, Madrid (2005)

LeCompte, M., Un matrimonio conveniente: diseño de investigación cualitativa y estándares para la evaluación de programas, RELIEVE, 1(1), 15-23 (1995)

Lincoln, Y. y Guba, E., Naturalistic Enquiry. Sage, Beverly Hills, California (1989)

McCabe, A. y O'Connor, U., Student-centred learning: the role and responsibility of the lecturer. Teaching in Higher Education, 19(4), 350-359 (2014)

Ministerio de Educación, Sistemas universitarios modernos: Diversidad y calidad institucional, Centro de Estudios Mineduc, 2 (16), 1-7 (2013)

Monereo, C, y Domínguez, C., La identidad docente de los profesores universitarios competentes, Educación XX1, 17(2), 83-104 (2014)

Moreno, I.R., Formación y profesionalización de la docencia en el nivel superior en el contexto de la posmodernidad. Reflexiones sobre el caso mexicano. Revista Iberoamericana de Evaluación Educativa, 3(1e), 203-217 (2016)

Morán, R., Cardoso, E., Cerecedo, M., y Ortiz, J., Evaluación de las competencias docentes de profesores formados en instituciones de educación superior: El caso de la asignatura de Tecnología en la Enseñanza Secundaria, Revista Formación Universitaria, 8(3), 57-64 (2015)

Ortega, M. C., Competencias Emergentes del Docente ante las Demandas del Espacio Europeo de Educación Superior, Revista Española de Educación Comparada, 16, 305-327 (2010)

Perrenoud, P., Diez nuevas competencias para enseñar, Graó, Barcelona (2004)

Postareff, L. y Lindblom- Ylänne, S., Variation in teachers' description of teaching: Broadening the understanding of teaching in higher education, Learning and Instruction, 18, 109-120 (2008)

Salgado, F., Corrales, J., Muñoz, L. y Delgado, J., Diseño de programas de asignaturas basados en competencias y su aplicación en la Universidad del Bío-Bío, Chile, Ingeniare, Revista Chilena de Ingeniería, 20(2), 267-278 (2012)

SEMS, Competencias que expresan el perfil del docente de la educación media superior. Documento Interno. Subsecretaría de Educación Media Superior. SEP, México (2008)

Slavich, G. y Zimbardo, P., Transformational Teaching: Theoretical Underpinnings, Basic Principles, and Core Methods. Educ. Psychol. Rev., 24, 569- 608 (2012)

Tejedor, F. J., Evaluación del Desempeño Docente. Revista Iberoamericana de Evaluación Educativa: 5(1e), 318-327 (2012)

Torelló, Ó. M., Las competencias del docente universitario: la percepción del alumno, de los expertos y del propio protagonista, Revista de Docencia Universitaria, 10(2), 299-318 (2012)

Torra, I., de Corral, I. y otros 17 autores, Identificación de competencias docentes que orienten el desarrollo de planes de formación dirigidos a profesorado universitario, Revista de Docencia Universitaria, 10(2), 21-56 (2012)

Triadó, X., Estebanell, M., Màrquez, M. D., y del Corral, I., Identificación del perfil competencial docente en educación superior. Evidencias para la elaboración de programas de formación continua del profesorado universitario, Revista Española de Pedagogía, 257, 51-72 (2014)

Zabalza, M. A., Ser un profesor universitario hoy, La Cuestión Universitaria, 5, 69-81 (2009) 
